\title{
Evaluation of surface cracks of bending concrete using a fully non-contact air-coupled nonlinear ultrasonic technique
}

\author{
Jun Chen · Chenglong Yang • Quanquan Guo
}

Received: 23 April 2018/Accepted: 16 July 2018/Published online: 20 July 2018

(C) The Author(s) 2019, corrected publication April 2019

\begin{abstract}
In this paper, a fully non-contact second harmonic generation (SHG) technique using a pair of air-coupled ultrasonic transducers is developed and the feasibility of the technique is investigated through the evaluation of surface cracks of concrete beams subjected to the bending load. The reliability of developed technique is subsequently validated by comparing the non-contact nonlinear ultrasonic measurements with measurement results based on contacttype sensors, where the coefficient of variation of noncontact measurements is averagely about $46 \%$ of the contact measurements. The defined nonlinear parameter is found to have a monotonically increasing trend with the growth of concrete crack, and the nonlinear parameter corresponding to the largest crack increases about 7 times from its initial value corresponding to the sample in intact state. In contrast, the increase of linear parameter namely the time-of-flight of surface wave is only about $40 \%$. The difference as high as one order of magnitude verifies the high sensitivity of developed air-coupled SHG technique. With consideration of the easily removable characteristic of air-coupled
\end{abstract}

The original version of this article was revised due to a retrospective Open Access order.

J. Chen · C. Yang · Q. Guo (ه)

Department of Civil Engineering, School of

Transportation Science and Engineering, Beihang

University, 37 Xueyuan Road, Haidian District,

Beijing 100191, China

e-mail: qq_guo@buaa.edu.cn ultrasonic measurements, the developed SHG technique could be promising for the large scale quality control of concrete structures in engineering practice.

Keywords Nonlinear ultrasonic $\cdot$ Air-coupled transducer $\cdot$ Rayleigh waves $\cdot$ Second harmonic generation $\cdot$ Concrete crack

\section{Introduction}

The evaluation of health condition of aged concrete structures is of importance for the service security. Various nondestructive evaluation (NDE) techniques have been attempted to assess the damage of concrete structures and experimental techniques based on the ultrasonic wave propagation are the most commonly used in academia and industry [1-5]. Particularly in recent years, a new group of ultrasonic methods in the light of nonlinear ultrasonic theory have gain substantial attentions due to their highly sensitive response to the structural defects in a variety of materials [6-9].

There exist several types of nonlinear ultrasonic techniques according to the different ultrasonic phenomena. The first method is based on the investigation of higher order harmonic of primary signal, which could be excited due to the existence of structural damage. For example, Nucera and Lanza di Scalea [10] used the second harmonic generation technique to monitor load levels in multi-wire steel strands in concrete. The second method is based on the 
examination of extra sidebands due to wave modulation phenomena when a low-frequency and highfrequency signal interact with each other. Wu et al. [11] and Chen et al. [12] employed the impact vibration signal and continuous harmonic signal as the low-frequency source to modulate the highfrequency wave for the damage characterization of concrete samples. The third method is the analysis of resonance frequency shift when the self-resonance of structures is excited by the external trigger. Payan et al. [13] and Chen et al. [14] developed the nonlinear ultrasonic resonance spectroscopy and nonlinear impact resonance acoustic spectroscopy to investigate the thermal and mechanical damage of concrete. Although these nonlinear ultrasonic techniques showed the excellent feasibility for damage assessment of concrete in different application cases, the experimental set up was mainly dependent of contacttype sensors and the coupling condition between sensors and sample surface has a significant influence on the reliability of signal measurements. The reliability problem of ultrasonic methods using contacttype sensors greatly limit their applications in engineering practice, where the large-scale inspection area of concrete structures inevitably requires the reinstallation of sensors at different inspection locations.

To reduce the effect of contact coupling and improve the measurement reliability, researchers began to explore the application possibility of aircoupled transducers in the nonlinear ultrasonic techniques. Thiele et al. [15] developed a half non-contact SHG technique for the material damage assessment where a wedged regular transducer is used as the signal transmitter and an air-coupled transducer is used as the receiver. Kim et al. [16, 17] further evaluated the variation of internal porosity of concrete due to the effect of carbonation and shrinkage using the developed half non-contact SHG technique. In et al. [18] developed a fully non-contact ultrasonic setup to measure the linear transmission coefficient of surface wave across a surface breaking crack in concrete. In addition, Maier [19] proposed a noncontact nonlinear resonance ultrasound spectroscopy for the analysis of microscopic damage of small metallic samples. However, it has to be noted that the hysteresis nonlinearity is measured in the nonlinear resonance ultrasound spectroscopy and is different from the quadratic nonlinearity measured in the air-coupled SHG technique.
In this paper, a fully non-contact experimental setup is developed as a further progress of the half noncontact SHG method, where both transmitting and receiving transducers are air-coupled sensors. The fully non-contact SHG technique is used to characterize the concentrated crack of reinforced concrete beam subjected to the bending load. The feasibility of the proposed experimental technique is firstly validated by checking the proportional relationship between the second harmonic amplitude and square of the primary signal amplitude. The measurement reliability is then examined by a comparison of coefficient of variation of multiple measurements based on the contact sensors and non-contact sensors. Finally, the variation of nonlinear parameter corresponding to different bending cracks is investigated and compared with the linear parameter such as the time-of-flight (TOF) of surface waves for the validation of sensitivity of the developed technique.

\section{Theoretical background of nonlinear parameter measurements}

As described by the nonlinear acoustic theory [20], the cracks of concrete could produce both the classical nonlinearity expressed by the power series of the strain and the nonclassical hysteresis nonlinearity containing the term of strain rate. The even harmonic is only caused by the classical nonlinearity and the odd harmonic is caused by both the classical and nonclassical nonlinearity [20]. Since only the second harmonic is measured in this work, the material nonlinearity is assumed to solely come from the quadratic order of strain in the constitutive relationship [21, 22]. The measurement of quadratic nonlinear parameter is thus the key step for the nonlinear ultrasonic characterization of material defects. As follows, the theoretical background of nonlinear parameter measurements based on the Rayleigh surface wave is concisely presented and more details can be referred to previous publications [23-26].

First, the Rayleigh wave is assumed to propagate along the $x$ axis of an isotropic, macroscopically homogeneous, and nonlinear elastic half space and $z$ axis refers to the distance to the surface.

Because of the existence of the shear and longitudinal wave, the linear displacement in $z$ axis can be expressed as [23] 
Table 1 Mixture proportioning of concrete sample $\left(\mathrm{kg} / \mathrm{m}^{3}\right)$

\begin{tabular}{llllll}
\hline Composition & Cement & Water & $w / c$ ratio & Fine sand & Aggregate \\
\hline Quantity & 490 & 220 & 0.45 & 590 & 1100 \\
\hline
\end{tabular}

$u_{z}(\omega)=i A_{1} \frac{b_{1}}{k_{R}}\left(e^{b_{1} z}-\frac{2 k_{R}^{2}}{k_{R}^{2}+b_{2}^{2}} e^{b_{2} z}\right) e^{i\left\{k_{R}\left(x-C_{R} t\right)\right\}}$

where $C_{R}$ is the speed of the Rayleigh wave, $\omega=C_{R} k_{R}$ is the fundamental frequency, $b_{1}=\sqrt{k_{R}^{2}-k_{L}^{2}}$ and $b_{2}=\sqrt{k_{R}^{2}-k_{S}^{2}} \cdot k_{L}, k_{S}$ and $k_{R}$ are the wave numbers of the longitudinal wave, the shear wave and the Rayleigh wave.

Analogously, we can calculate the displacement of the second harmonic as [22, 23]

$u_{z}(2 \omega)=i A_{2} \frac{b_{1}}{k_{R}}\left(e^{b_{1} z}-\frac{2 k_{R}^{2}}{k_{R}^{2}+b_{2}^{2}} e^{b_{2} z}\right) e^{i\left\{k_{R}\left(x-C_{R} t\right)\right\}}$

It has been demonstrated that the quadratic nonlinear parameter is related to the out-of-plane displacement of the Rayleigh waves at the surface $(z=0)$ as shown in Eq. (3) [22]

$\beta=\frac{\left.u_{z}(2 \omega)\right|_{z=0}}{\left.u_{z}^{2}(\omega)\right|_{z=0}} \frac{8 b_{i} i}{k_{1}^{2} k_{R} x}\left(1-\frac{2 k_{R}^{2}}{k_{R}^{2}+b_{2}^{2}}\right)$

Combine Eqs. (1), (2) and (3), then simplify the equation, the relationship of $A_{1}, A_{2}$ and $\beta$ can be obtained as follows [17]

$\beta \propto \frac{A_{2} x}{A_{1}^{2}}$

Since the propagating distance $x$ is constant in the SHG measurements of this work, the nonlinear parameter $\beta$ used in this work is expressed as

$\beta=\frac{A_{2}}{A_{1}^{2}}$

\section{Experimental procedure}

\subsection{Specimen}

A reinforced concrete specimen $(100 \times 100 \times$ $400 \mathrm{~mm}$ ) was prepared for the experiment. River gravel (maximum size of $20 \mathrm{~mm}$ ) as coarse aggregate, ordinary sand (maximum size of $2 \mathrm{~mm}$ ) as fine aggregate, Type $\mathrm{P} \bullet \mathrm{O} 42.5$ (ordinary Portland cement with a nominal strength of $42.5 \mathrm{MPa}$ ) and water were used for the cast of concrete specimen, and a water-tocement ratio $(w / c)$ of 0.45 was designed in the mixing. Two steel bars with a diameter of $8 \mathrm{~mm}$ were placed near the bottom of specimen to mitigate the brittle failure during the bending tests. Table 1 shows the detailed mix proportion of the concrete sample. The specimen was cured in a standard curing chamber $\left(23{ }^{\circ} \mathrm{C}\right.$ and $95 \%$ relative humidity) for 28 days after demolding.

\subsection{Bending test of the concrete beam}

The concrete damage was induced by the three-point bending test, where the sample was placed on a universal loading machine and an increased bending load was applied on the middle spot of the top surface of concrete sample. For each loading level, the sample was pull out from the loading frame for the ultrasonic experiments and crack width measurements. A crack detector was installed near the middle spot of bottom surface to record the images of bending crack. The image of cracks at different loading stage is shown in Fig. 1, where a clear growth of crack width due to the increasing bending load is seen while the crack length keeps the same.

\subsection{Experimental setup of air-coupled SHG measurements}

The experimental setup of air-coupled SHG measurements is shown in Fig. 2. A tone-burst signal at a frequency of $50 \mathrm{kHz}$ is first generated by a digital function generator (Rigol DG1022). A power amplifier (Krohn-Hite 7500) is then used to amplify the signal by a gain of $40 \mathrm{~dB}$ which is then fed into an aircoupled transducer (Ultran NCG50-D50) having a central frequency of $50 \mathrm{kHz}$. The Rayleigh wave transmits through the concrete specimen and is picked up by the second air-coupled transducer (Ultran 

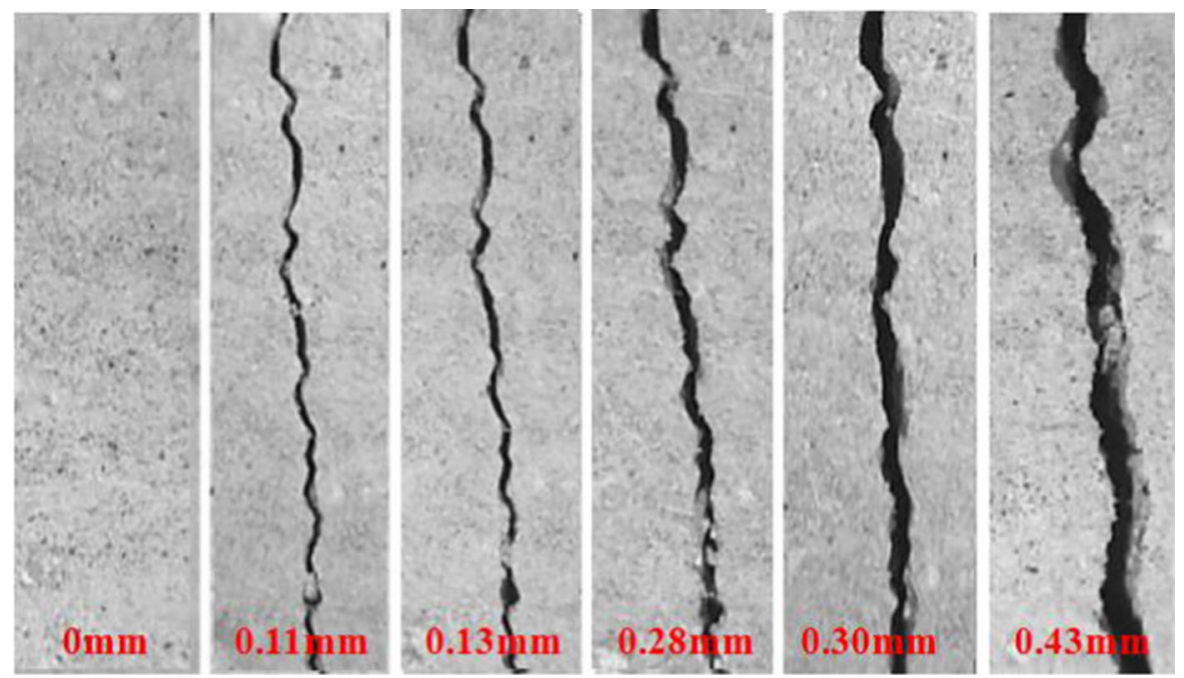

Fig. 1 Image of cracks at the different bending load

Fig. 2 Experimental setup of fully air-coupled SHG measurements

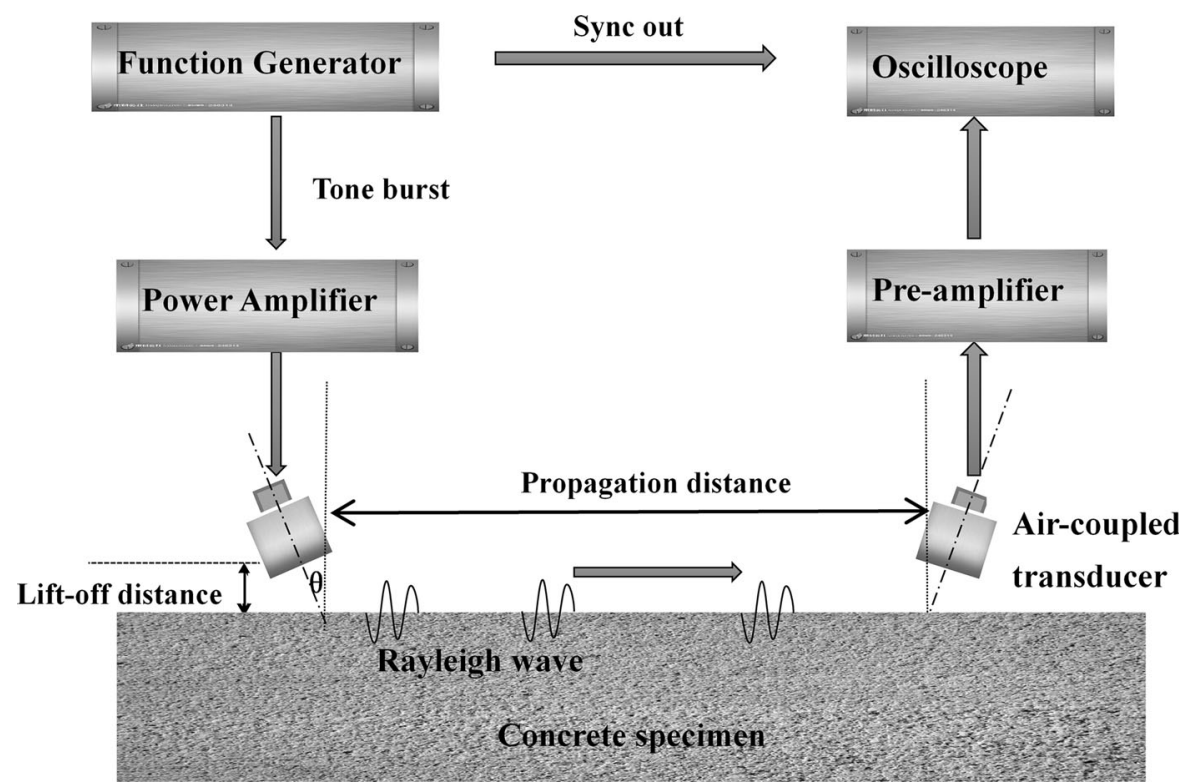

NCG100-D50) having a central frequency of $100 \mathrm{kHz}$. The peak-to-peak voltage of the signal changes from $15 \mathrm{~V}$ to $20 \mathrm{~V}$ with a step of $0.5 \mathrm{~V}$. The received signal is averaged 64 times and eventually recorded by a digital oscilloscope (Tektronix MDO3012).

The phase velocity of the Rayleigh wave in the concrete specimen is needed for the determination of incidence angel of transducers, and it is measured using a high power pulser-reciever (Olympus 5077PR) in experiments. A pair of regular ultrasonic transducers were glued to wedges made of Teflon and firmly attached with the specimen surface for the measurement of traveling time of different propagation distance, and the phase velocity can be calculated accordingly. With knowing the phase velocity, the critical incidence angle for the Rayleigh wave $\theta$ (i.e., the tilted angle of the central axis of the air-coupled transducer from the plane perpendicular to the concrete surface) is calculated according to Snell's law, which is set to be $11^{\circ}$ in experiments. The vertical 
distance between the central location of air-coupled transducers and the concrete surface (the lift-off distance) is designed to be $15 \mathrm{~mm}$ to ensure a satisfactory signal strength.

\section{Results and discussion}

\subsection{Determination of nonlinear parameter}

Figure 3 shows a time-domain signal obtained in experiments, which may contain multiple reflections since the length of tone burst signal is larger than the sample length. As theoretically proved in one author's recent work [27], the defined nonlinear parameter $\beta$ originally for a propagating wave can be measured also from the second harmonic generation in standing waves. A Hanning window is applied on the timedomain signal and the selection criterion of window size and location is to get the steady part of propagating wave. The same window size and location is kept for all ultrasonic signals and thus the relative comparison of nonlinear parameter will not be affected by the window selection. The spectra of the windowed signals with the increased input amplitude are obtained in Fig. 4 using the fast Fourier transform (FFT). The FFT magnitudes of primary signal and second harmonic in the spectra of Fig. 4 are defined as $M_{1}$ and $M_{2}$ respectively and are used to represent the amplitude $A_{1}$ and $A_{2}$ in Eq. (5) since the frequencydomain magnitude is proportional to the time-domain amplitude. The nonlinear parameter $\beta^{\prime}$ is then

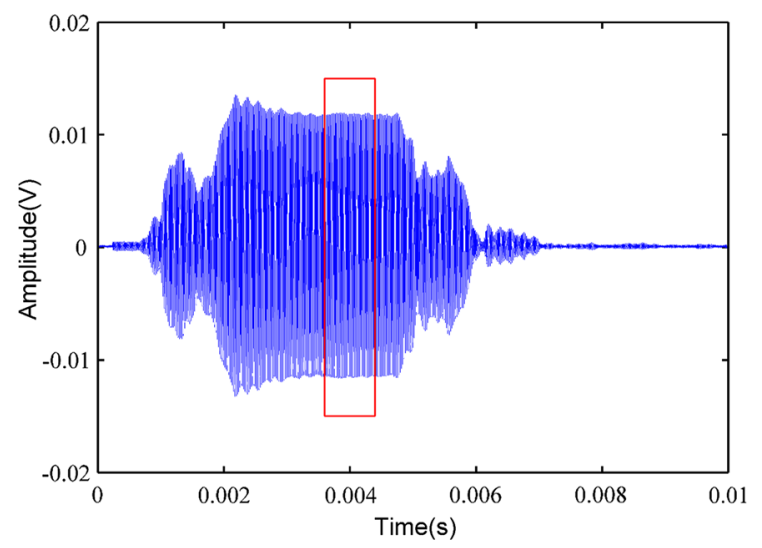

Fig. 3 Time-domain signal of fully air-coupled SHG measurements

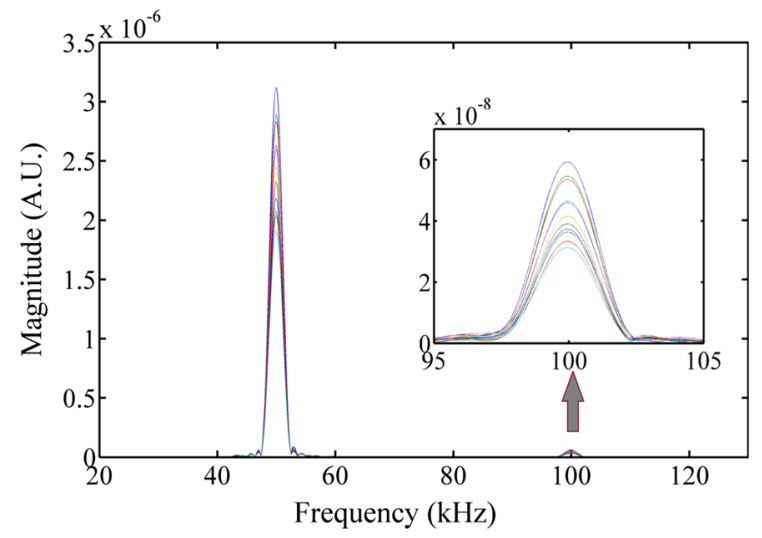

Fig. 4 Frequency-domain signal of fully air-coupled SHG measurements

calculated by the slope of linear relationship between $M_{1}^{2}$ and $M_{2}$ as shown in Fig. 5.

In addition, it has to be noted that the instruments themselves may generate spurious nonlinearity to affect the nonlinearity measurement of concrete sample. To further validate the instrument nonlinearity negligible, a known linear sample - a PMMA plate is used in the air-coupled second harmonic experiments. The experimental setup and procedure is exactly the same as for the concrete sample. The nonlinear parameter of the PMMA plate calculated is only $4.6 \%$ of the value of intact concrete. Thus, the instrumentation nonlinearity is demonstrated to have little influence compared to crack nonlinearity.

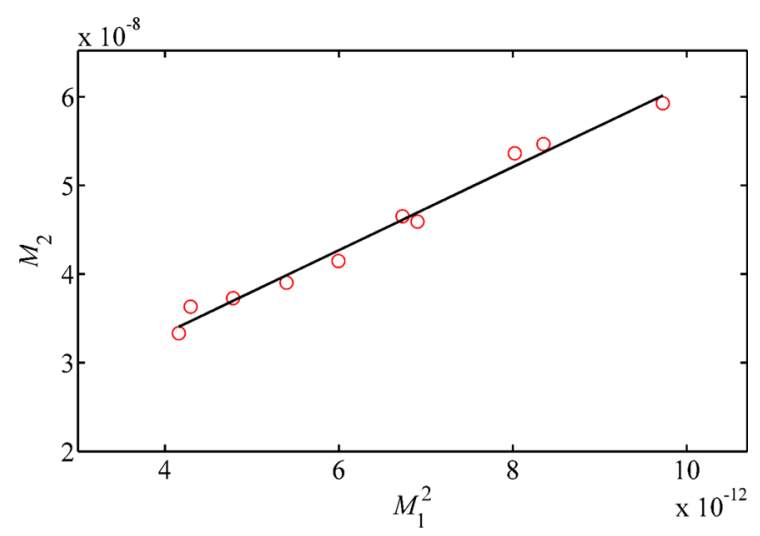

Fig. 5 Nonlinear parameter $\beta^{\prime}$ obtained from the linear relationship of $M_{1}^{2}$ and $M_{2}$ 


\subsection{Results of phase velocity of Rayleigh wave}

The phase velocity of Rayleigh wave is considered as a typical parameter to characterize the structure defects and is thus used here for a comparison with the nonlinear parameter of SHG measurements. The variation of phase velocity of Rayleigh wave is plotted in Fig. 6. It is seen the phase velocity generally has a decreasing trend with the development of bending crack and the maximal decrease corresponding to the largest crack width is about $27 \%$. However, the velocity does not present a substantial change when the crack is tiny. The decrease of velocity from the intact state to the crack width of $0.11,0.13$ and $0.28 \mathrm{~mm}$ is about $1.2,7.9$ and $11.0 \%$, respectively. The results in Fig. 6 indicate that the phase velocity of Rayleigh wave is not very sensitive to the early phase of bending crack development, which is consistent with previous experimental observations based on bulk waves [28].

\subsection{Nonlinear SHG measurements}

The SHG measurements were conducted after each loading and the nonlinear quadratic parameter was calculated following the procedure described above. The linear relationship between $M_{2}$ and $M_{1}^{2}$ at different crack width is shown in Fig. 7, where the values of both axis were normalized to make all data distribute in the similar range. An excellent coefficient of correlation is generally seen for each line and the slope has a clear increasing trend. The results in Fig. 7 indicate that the SHG measurements based on aircoupled transducers can well characterize the material

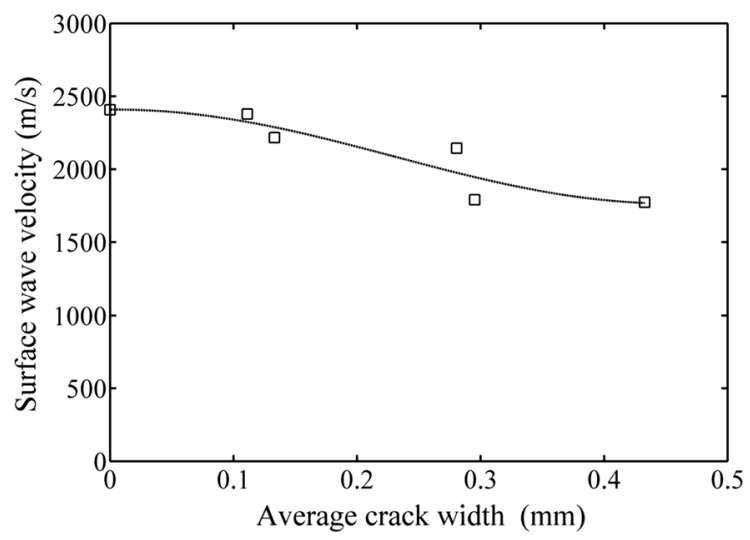

Fig. 6 Variation trend of phase velocity of Rayleigh wave with respect to average crack width

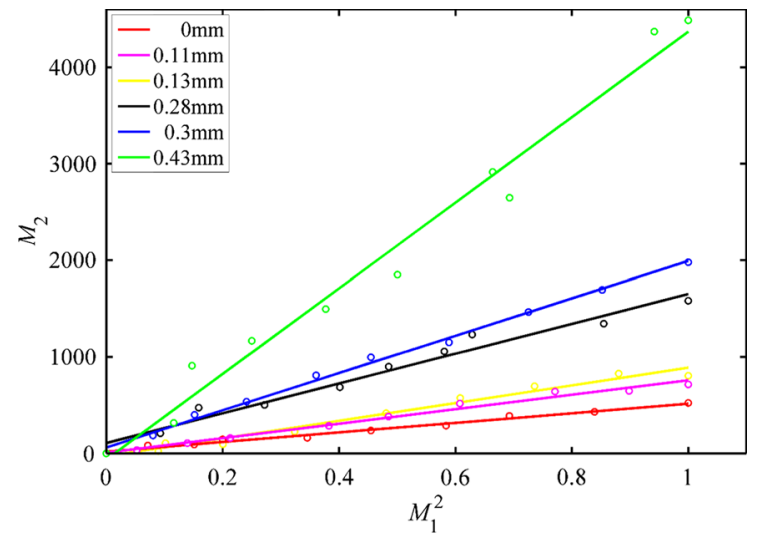

Fig. 7 Variation of $M_{1}^{2}$ and $M_{2}$ of concrete specimen with increased crack width

nonlinearity due to bending crack. The normalized nonlinear parameter is plotted in Fig. 8, where a rapid growth is seen throughout the entire course of bending test. For instance, the nonlinear parameter of the crack width of $0.13 \mathrm{~mm}$ increases about $72 \%$ from the intact state of specimen, and the nonlinear parameter further grows about 2.7 times when the crack develops to a width of $0.30 \mathrm{~mm}$. The error bars in Fig. 8 refer to the standard deviation arising from the three measurements where the transducers were re-located at the same spot for each time.

\subsection{Reliability and sensitivity analysis}

To validate the reliability of SHG measurements using air-coupled transducers, the contact-type SHG

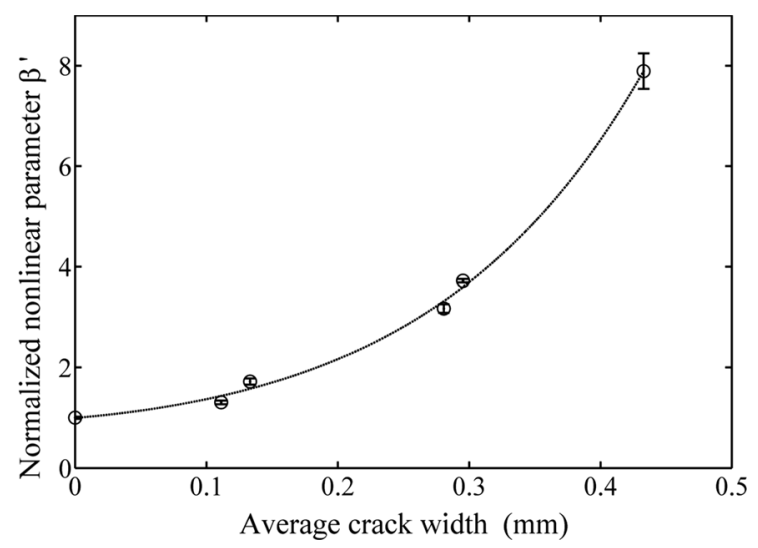

Fig. 8 Variation trend of nonlinear parameter $\beta^{\prime}$ with respect to average crack width in the case of non-contact SHG measurements 
measurements were conducted simultaneously for the same specimen, where two wedged ultrasonic transducers were used. The measurements for each crack width were repeated three times where the wedges were removed and reattached to the same locations of the specimen. The normalized nonlinear parameter of the contact-type SHG measurements is plotted in Fig. 9. Through a comparison of Figs. 8 and 9, it is seen that the nonlinear parameters from contact measurements and non-contact measurements have an excellent linear correlation where the coefficient of correlation is as high as 0.986. Furthermore, the coefficient of variation of contact measurements and non-contact measurements for each crack state is compared in Fig. 10. The average level of coefficient of variation of non-contact measurements is about $46 \%$ of that of contact measurements, showing that the reliability of air-coupled SHG measurements is better than the contact SHG measurements.

Since the phase velocity decreases with the crack growth and this trend is opposite to the variation of nonlinear parameter, it is necessary to convert the phase velocity to a physical parameter having the increasing trend for the better demonstration of sensitivity comparison between SHG measurements and linear measurements. With consideration that the propagating distance of surface wave is known, the TOF of surface wave calculated by dividing the propagating distance by the phase velocity is used to compare with the nonlinear parameter. The variations of TOF of surface wave and nonlinear parameter are plotted in Fig. 11. The increase of nonlinear parameter

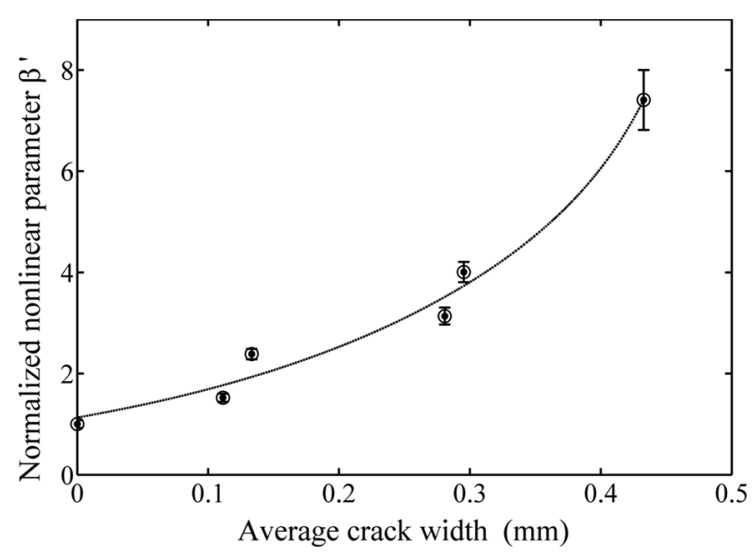

Fig. 9 Variation trend of nonlinear parameter $\beta^{\prime}$ with respect to average crack width in the case of contact SHG measurements

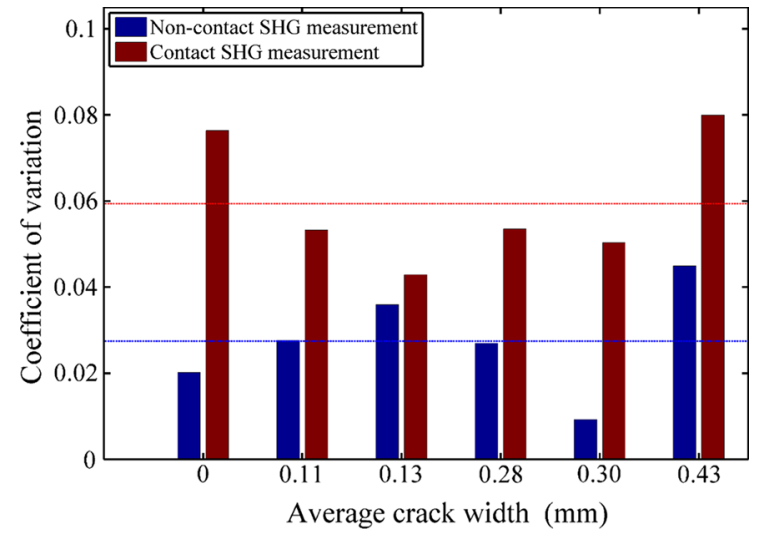

Fig. 10 Comparison of coefficient of variation for non-contact and contact SHG measurements

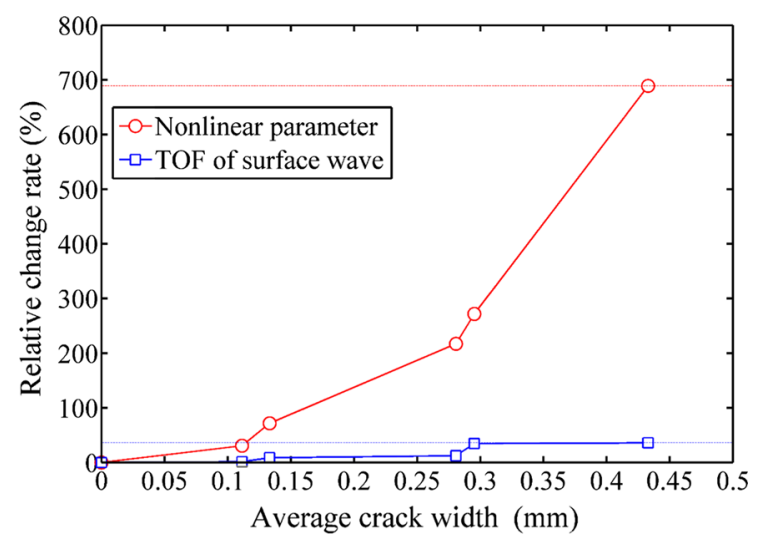

Fig. 11 Comparison of increasing trend of nonlinear parameter and linear parameters

shows a very large distinction from the increase of TOF of surface wave during the entire course of crack occurrence and growth. The maximal increase of TOF of surface wave corresponding the largest crack is about $40 \%$ while the maximal increase of nonlinear parameter is about $700 \%$. The sensitivity of SHG measurements based on air-coupled transducers is thus validated.

\section{Conclusion}

A fully non-contact SHG technique based on both aircoupled transmitting and receiving transducers is developed in this paper and is applied for the crack evaluation of the reinforced concrete beam subjected 
to the bending load. The conclusions of this paper are as follows:

1. The fully air-coupled SHG technique is feasible for the detection of bending crack in the concrete specimen. Pronounced second harmonics are generated with the propagating Rayleigh wave across the crack and the variation of second harmonic amplitude with respect to the square of primary signal amplitude conforms to the linear trend predicted by the theoretical derivation of nonlinear ultrasonic theory.

2. The developed experimental technique has a better reliability compared to contact-type SHG method. The coefficient of variation of multiple measurements using air-coupled transducers is about $46 \%$ of the coefficient of variation of SHG measurements using contact transducers.

3. The developed technique has a better sensitivity in terms of crack detection compared to the conventional phase velocity method. The nonlinear parameter has a relative increase as high as $700 \%$ from the intact state of concrete specimen while the change of TOF of surface wave is about $40 \%$.

Based on the current finding of this work, the fully non-contact SHG technique demonstrates to be an effective method for the crack characterization of concrete owing to its feasibility, stability and sensitivity. Furthermore, the non-contact characteristic of this technique make it convenient for the repeatable measurements on a large scale area since no reinstallation of transducers is needed. Combing with the automatic moving and relocating system, the developed technique is promising for the rapid quality control of large concrete structures on site.

Acknowledgements The authors gratefully acknowledge the support from National Natural Science Foundation of China (Grant Nos. 51308020 and 51578032), Major Fundamental Research of China (973 Program, Grant No. 2014CB047003) and Beijing Natural Science Foundation (Grant No. 8162027). The effort made by Mr. Qingdong Wang in part of the experimental work is appreciated.

\section{Compliance with the ethical standards}

Conflict of interest All authors declare that they have no conflict of interest.
Open Access This article is distributed under the terms of the Creative Commons Attribution 4.0 International License (http:// creativecommons.org/licenses/by/4.0/), which permits use, duplication, adaptation, distribution and reproduction in any medium or format, as long as you give appropriate credit to the original author(s) and the source, provide a link to the Creative Commons license and indicate if changes were made.

\section{References}

1. Arizzi A, Martínez-Martínez J, Cultrone G (2013) Ultrasonic wave propagation through lime mortars: an alternative and non-destructive tool for textural characterization. Mater Struct 46:1321-1335

2. Chung CW, Suraneni P, Popovics JS, Struble LJ, Weiss WJ (2013) Application of ultrasonic p-wave reflection to measure development of early-age cement-paste properties. Mater Struct 46:987-997

3. Zhang J, Liu C, Sun M, Li Z (2017) An innovative corrosion evaluation technique for reinforced concrete structures using magnetic sensors. Constr Build Mater 135:68-75

4. Ohtsu M (2011) Damage evaluation in freezing and thawing test of concrete by elastic-wave methods. Mater Struct 44:1725-1734

5. Zhou C, Li K, Han J (2012) Characterizing the effect of compressive damage on transport properties of cracked concretes. Mater Struct 45:381-392

6. Zaitsev VY, Matveev LA, Matveyev AL (2011) Elasticwave modulation approach to crack detection: comparison of conventional modulation and higher-order interactions. NDT\&E Int 44:21-31

7. Leśnicki KJ, Kim J-Y, Kurtis KE, Jacobs LJ (2011) Characterization of ASR damage in concrete using nonlinear impact resonance acoustic spectroscopy technique. NDT\&E Int 44:721-727

8. Shui GS, Wang YS, Huang P, Qu JM (2015) Nonlinear ultrasonic evaluation of the fatigue damage of adhesive joints. NDT\&E Int 70:9-15

9. Shui GS, Wang YS, Gong F (2013) Evaluation of plastic damage for metallic materials under tensile load using nonlinear longitudinal waves. NDT\&E Int 55:1-8

10. Nucera C, Scalea FLD (2011) Monitoring load levels in multi-wire strands by nonlinear ultrasonic waves. Struct Health Monit 10:617-629

11. Warnemuende K, Wu H-C (2004) Actively modulated acoustic nondestructive evaluation of concrete. Cem Concr Res 34:563-570

12. Chen J, Jayapalan AR, Kim J-Y, Kurtis KE, Jacobs LJ (2010) Rapid evaluation of alkali-silica reactivity of aggregates using a nonlinear resonance spectroscopy technique. Cem Concr Res 40:914-923

13. Payan C, Garnier V, Moysan J, Johnson PA (2007) Applying nonlinear resonant ultrasound spectroscopy to improving thermal damage assessment in concrete. J Acoust Soc Am 121:EL125 
14. Chen J, Zhang L (2015) Experimental study of effects of water-cement ratio and curing time on nonlinear resonance of concrete. Mater Struct 48:423-433

15. Thiele S, Kim J-Y, Qu J, Jacobs LJ (2014) Air-coupled detection of nonlinear Rayleigh surface waves to assess material nonlinearity. Ultrasonics 54:1470-1475

16. Kim G, In C-W, Kim J-Y, Kurtis KE, Jacobs LJ (2014) Aircoupled detection of nonlinear Rayleigh surface waves in concrete-application to microcracking detection. NDT\&E Int 67:64-70

17. Kim G, Kim J-Y, Kurtis KE, Jacobs LJ, Pape Y, Guimaraes M (2016) Quantitative evaluation of carbonation in concrete using nonlinear ultrasound. Mater Struct 49:399-409

18. In C-W, Schempp F, Kim J-Y, Jacobs LJ (2014) A fully noncontact, air-coupled ultrasonic measurement of surface breaking cracks in concrete. J Nondestr Eval 34:272

19. Maier S (2017) Noncontact nonlinear resonance ultrasound spectroscopy for small metallic samples. Master Thesis, Georgia Institute of Technology

20. Van Den Abeele KE-A, Johnson PA, Sutin A (2000) Nonlinear elastic wave spectroscopy (NEWS) techniques to discern material damage, Part I: nonlinear wave modulation spectroscopy (NWMS). Res Nondestr Eval 12:17-30

21. Landau LD, Lifshitz EM (1986) Theory of elasticity, 3rd edn. Butterworth-Heinemann, Oxford
22. Guyer RA, Johnson PA (1999) Nonlinear mesoscopic elasticity: evidence for a new class of materials. Phys Today 52:30-35

23. Viktorov IA (1967) Rayleigh and lamb waves. Plenum Press, New York

24. Herrmann J, Kim J-Y, Jacobs LJ, Qu J, Littles JW, Savage MF (2006) Assessment of material damage in a nickel-base super alloy using nonlinear Rayleigh surface waves. J Appl Phys 99:1479-1488

25. Matlack KH, Kim J-Y, Jacobs LJ, Qu J (2015) Review of second harmonic generation measurement techniques for material state determination in metals. J Nondestr Eval 34:273

26. Torello D, Thiele S, Matlack KH, Kim J-Y, Qu J, Jacobs LJ (2015) Diffraction, attenuation, and source corrections for nonlinear Rayleigh wave ultrasonic measurements. Ultrasonics 56:417-426

27. Chen J, Yin TY, Kim J-Y, Xu Z, Yao YP (2017) Characterization of thermal damage in sandstone using the second harmonic generation of standing waves. Int J Rock Mech Min Sci 91:81-89

28. Chen J, Ren J, Yin T (2015) Nondestructive evaluation of notched cracks in mortars by nonlinear ultrasonic technique. Nondestr Test Eval 31:109-121 